\title{
Usefulness of Trans-Thoracic Echocardiography (TTE) in patients with acute ischaemic strokes and normal clinical cardiac examination
}

\author{
Dilhani PDW, Lekamwasam S \\ University Medical Unit, Teaching Hospital Karapitiya, Galle, Sri Lanka. \\ Correspondence: Dr. PDW Dilhani \\ e-mail: warshadez@gmail.com
}

\begin{abstract}
Introduction: Stroke is one of the leading causes of disability and mortality in Sri Lanka. Cardiac sources of embolism account for $15-30 \%$ of ischaemic strokes. For optimal treatment aiming at secondary prevention it is crucial to identify the source of the embolism. We aimed to assess the influence of routine echocardiography in the management of patients with ischaemic strokes who have no evidence of cardiac abnormality on routine medical history, physical examination or 12-lead ECG.

Methods: Transthoracic echocardiography (TTE) was performed in patients admitted to Professorial Medical Unit, Teaching Hospital Karapitiya with an acute ischaemic stroke and normal clinical cardiac evaluation including a 12-lead ECG. The echo findings were categorized as therapeutically significant or insignificant by the cardiologist who performed the echo and the treating physician.

Results: Of the 110 patients with acute ischaemic stroke with normal clinical cardiac evaluation, $14.5 \%$ had therapeutically significant echo findings; left ventricular systolic dysfunction with ejection fraction $<35 \%$ in 13 $(11.8 \%)$, left ventricular aneurysm with intraventricular clot in $2(1.8 \%)$, and tight mitral stenosis in $1(0.9 \%)$.

Conclusions: In this study, TTE revealed cardiac abnormalities that need specific treatment in $16(14.5 \%)$ patients with acute ischaemic stroke with normal clinical cardiac evaluation. TTE should be considered an essential test in the evaluation of all ischaemic stroke patients regardless of the clinical findings.
\end{abstract}

Key Words: Transthoracic echocardiography, ischaemic stroke, Sri Lanka.

\section{Introduction}

Stroke is one of the leading causes of disability and mortality in Sri Lanka. Ischaemic stroke accounts for about $70-80 \%$ of all strokes and is caused by embolic or thrombotic occlusions in the cerebral vessels (1). Large artery atherosclerosis is the commonest aetiology of ischaemic strokes. Further, cardiac sources of embolism account for $15-30 \%$ of the cases (1). The latter is associated with poor prognosis and fatal recurrences. In order to establish an optimal preventive strategy it is crucial to identify the source of the embolism.
To find the cardiac source of embolism a detailed medical history and physical examination are required. In addition, ECG and chest radiograph are routine investigations performed in order to find cardiac abnormalities which can be potential sources of embolization. There is a considerable disagreement, however, among clinicians regarding the extent of cardiac testing that should be performed in patients with ischaemic stroke, especially in less resourceful settings (2).

The routine use of echocardiography in all patients with ischaemic strokes is debatable, particularly when the detailed history, physical examination and 
ECG fail to show any evidence of previous or ongoing cardiac pathology (2). Furthermore, routine echo may show the existence of cardiac abnormalities which are unlikely to be the cause of ischaemic stroke, hence have no therapeutic implications. They include degenerative changes in the cardiac valves especially mitral and aortic valves.

A recent hospital based study from Canada involving patients with ischaemic strokes reports that TTE has a low yield, diagnosing only $4 \%$ of significant cardiac abnormalities (2). A similar single centre study from Pakistan revealed $15.5 \%$ patients to have TTE abnormalities suggestive of cardioembolic aetiology (3). We were unable to find previous studies with similar objectives done in Sri Lanka.

Therefore this study was planned to assess the usefulness of routine echocardiography in patients with ischaemic strokes who have no evidence of previous or ongoing cardiac abnormality on routine medical history, physical examination or 12-lead ECG.

\section{Methods}

This study was carried out in the Professorial Medical Unit of Teaching Hospital Karapitiya which is the largest hospital in the Southern province with the highest patient turnover. Consecutive patients with acute ischaemic strokes admitted to the above unit via outpatient department were included in the study. Medical Professorial Unit receives about 30 patients with acute ischaemic stroke per month.

All patients admitted with acute focal neurological deficit underwent urgent cranial CT imaging. All the CT images were examined for the presence of cerebral bleeding that can explain the clinical presentation. Those with normal CT images as well as those with $\mathrm{CT}$ evidence of recent cerebral ischaemia were considered to have cerebral infarctions and were eligible for this study. They all underwent routine vascular screening and TTE. For this analysis only those patients who had normal complete clinical cardiac evaluation (history and examination) and 12 lead ECG were considered. We included 110 consecutive patients for the study. An interviewer administered questionnaire was used as the study instrument. Cardiovascular examination, ECG and ECHO findings were also included in this questionnaire.
The echo findings were categorized as therapeutically significant (those needing further cardiac investigations or interventions) or therapeutically insignificant (age-related and those not needing further investigations or interventions). Categorization was done by caring physician in consultation with cardiologist who performed the TTE.

The ethical clearance for the study was obtained from the Ethics Committee Faculty of Medicine, University of Ruhuna, Sri Lanka.

\section{Results}

Out of 110 subjects in the sample, 60 (54.5\%) were males. Fifty eight $(52.7 \%)$ of patients were within $50-70$ year age range. Fourteen $(12.7 \%)$ were below 50 years and $38(34.6 \%)$ were above 70 years of age. Majority of the sample $(62.8 \%)$ belonged to either Social class 3 or 4 .

When risk factors were assessed, hypertension was the most common risk factor identified in this study. Seventy (63.6\%) had hypertension, 29 (26.4\%) had diabetes mellitus and 31 (28.2\%) had evidence of dyslipidaemia.

Thirty five (31 8\%) were smokers.

Past history of stroke and transient ischaemic attacks was observed in $9(8.2 \%)$ and $7(6.3 \%)$ of individuals, respectively. Twenty (18.2\%) participants had family history of ischaemic heart disease and 20 $(18.2 \%)$ had family history of stroke.

In the sample, 62 (56.4\%) had normal BMI while 47 $(42.7 \%)$ were either overweight or obese and only 1 (0.9\%) had low BMI.

Of 110 patients, CT evidence of cerebral infarction was evident in 69 (62.7\%) of individuals.

Therapeutically significant echocardiographic findings were detected in $16(14.5 \%)$ of study participants. They were, left ventricular ejection fraction $<35 \%$ in $13(11.8 \%)$, left ventricular aneurysm with a thrombus in $2(1.8 \%)$ and tight calcific mitral stenosis in $1(0.9 \%)$.

\section{Discussion}

In our study sample, $14.5 \%$ of patients with acute ischaemic stroke, with complete clinical 
cardiac evaluation, had therapeutically significant transthoracic echocardiographic findings: left ventricular systolic dysfunction with an ejection fraction $<35 \%(11.8 \%)$, left ventricular aneurysm with a thrombus $(1.8 \%)$ and tight calcific mitral stenosis $(0.9 \%)$.

A recent hospital-based study from Canada reports that transthoracic echocardiography has a low yield, diagnosing only $4 \%$ of ischaemic stroke patients with abnormalities suggesting cardio embolic aetiology (2). In contrast, another single centre study from Portugal reveals $37.2 \%$ of ischaemic stroke patients with transthoracic echocardiography abnormalities suggestive of cardio embolic aetiology and the authors recommended the use of echocardiography as a routine procedure in all stroke patients(1).

There was an $11.8 \%$ of patients with asymptomatic left ventricular systolic dysfunction with ejection fraction $<35 \%$ in our study. Literature review revealed that the prevalence of stage $B$ heart failure (reduced left ventricular ejection fraction without symptoms of heart failure) varied between $12.9 \%$ and $21.4 \%$ in the community and also increased with age $(4,5)$. Furthermore, diabetes and hypertension have been identified as the most important underlying causes of heart failure and they were prevalent among our study participants $(5,7)$. Tight calcific mitral stenosis is usually clinically inaudible and left ventricular aneurysm with an apical thrombus also cannot be diagnosed on clinical evaluation.

Current guidelines do not recommend transthoracic echocardiography as a routine test to evaluate patients with stroke with low risk of embolic aetiology. However, in this study, TTE findings changed the management plan of $14.5 \%$ of patients with acute ischaemic stroke with normal clinical cardiac evaluation. Therefore, we believe that these findings support the idea that transthoracic echocardiogram should be a compulsory investigation in the evaluation of all acute ischaemic stroke patients regardless of the clinical findings.

\section{References}

1. de Abreu TT, Mateus S, Correia J. Therapy implications of transthoracic echocardiography in acute ischaemic stroke patients. Stroke 2005; 36: 1565-6.

2. Douen A, Pageau N, Medic S. Usefulness of Cardiovascular Investigations in Stroke Management. Clinical Relevance and Economic Implications. Stroke 2007; 38: 1956-58. Epub 2007.

3. Muhib Alam Khan, Bhojo Khealani, Bilal Hameed. Diagnostic yield of transthoracic echocardiography for stroke patients in a developing country. JMPA 2008.

4. Azevedo A, Bettencourt P, Dias P, Abreu Lima C, Hense HW, Barros H. Population based study on the prevalence of the stages of heart failure. Heart. Aug 2006; 92(8): 1161-3.

5. Lee R. Goldberg, Mariell Jessup. Stage B Heart Failure. Circulation, 2006; 113: 2851-60.

6. Nancy M. Albert, Connie Lewis. Recognizing and Managing Asymptomatic Left Ventricular Dysfunction. Critical Care Nurse. April 2008; 28: 20-37.

7. Iung B, Gohlke C, Tornos P, Tribouilloy C, Hall R, Butchart E, Vahanian A. Recommendations on the management of the asymptomatic patient with valvular heart disease. European Heart Journal 2002; 23: 1253-66. 